\title{
Low-Temperature Rapid Fabrication of ZnO Nanowire UV Sensor Array by Laser-Induced Local Hydrothermal Growth
}

\author{
Sukjoon Hong, Junyeob Yeo, Wanit Manorotkul, Gunho Kim, \\ Jinhyeong Kwon, Kunsik An, and Seung Hwan Ko \\ Applied Nano Tech and Science Lab, KI for Nano Century, Graduate School of (Energy Environment Water and Sustainability) EEWS, \\ Korea Advanced Institute of Science and Technology (KAIST), 291 Daehak-ro, Yuseong-gu, Daejeon 305-701, Republic of Korea
}

Correspondence should be addressed to Seung Hwan Ko; maxko@kaist.ac.kr

Received 12 April 2013; Accepted 28 June 2013

Academic Editor: Naeimeh Naseri

Copyright (C) 2013 Sukjoon Hong et al. This is an open access article distributed under the Creative Commons Attribution License, which permits unrestricted use, distribution, and reproduction in any medium, provided the original work is properly cited.

We demonstrate $\mathrm{ZnO}$ nanowire based UV sensor by laser-induced hydrothermal growth of $\mathrm{ZnO}$ nanowire. By inducing a localized temperature rise using focused laser, $\mathrm{ZnO}$ nanowire array at $\sim 15 \mu \mathrm{m}$ size consists of individual nanowires with $\sim 8 \mu \mathrm{m}$ length and $200 \sim 400 \mathrm{~nm}$ diameter is readily synthesized on gold electrode within $30 \mathrm{~min}$ at the desired position. The laser-induced growth process is consecutively applied on two different points to bridge the micron gap between the electrodes. The resultant photoconductive $\mathrm{ZnO}$ NW interconnections display 2 3 orders increase in the current upon the UV exposure at a fixed voltage bias. It is also confirmed that the amount of photocurrent can be easily adjusted by changing the number of $\mathrm{ZnO} \mathrm{NW}$ array junctions. The device exhibits clear response to the repeated UV illumination, suggesting that this process can be usefully applied for the facile fabrication of low-cost UV sensor array.

\section{Introduction}

1D nanostructures, or nanowires (NWs), have been studied extensively for the future application in diverse areas including optoelectronics $[1,2]$, energy harvesting devices $[3,4]$, and sensors [5-9] due to its large surface-to-volume ratio and other unique properties. Among various materials, $\mathrm{ZnO} \mathrm{NW}$ can be employed usefully for sensor applications because of its high on/off ratio [10] and low cost fabrication [11], and the related studies reported that $\mathrm{ZnO} N W$ can detect the change in pressure [5], humidity [6], gas concentration [7], UV illumination [12-14], and even biomolecular interactions [8]. Menzel et al. also reported a $\mathrm{ZnO}$ NW based multifunctional sensor [9] which can detect temperature, UV exposure, and $\mathrm{pH}$ change in a single platform.

For the fabrication of conventional $\mathrm{ZnO} \mathrm{NW}$ based sensor having metal-semiconductor-metal (MSM) configuration, $\mathrm{ZnO} \mathrm{NW}$ is firstly grown in tube furnace by chemical vapor deposition (CVD) method [15] which requires a very high temperature of around $1000^{\circ} \mathrm{C}$. The grown $\mathrm{ZnO} \mathrm{NW}$ is then harvested and transferred to the target substrate, followed by e-beam lithography and evaporation [16] in order to form electrodes that capture two ends of the NW for further electrical characterization. These two aspectshigh temperature for the NW synthesis and difficult technique for capturing the NW with electrode layer-have been remained as big problems for the facile fabrication of $\mathrm{ZnO} \mathrm{NW}$ based sensors. Recent studies on $\mathrm{ZnO} \mathrm{NW}$ based sensor [12-14] attempt to utilize the $\mathrm{ZnO} \mathrm{NW}$ grown by hydrothermal method which can be conducted in very lowtemperature $\left(<100^{\circ} \mathrm{C}\right)$ compared to other CVD methods such as vapor-solid [17] (VS) or vapor-liquid-solid [18] (VLS); yet hydrothermally grown $\mathrm{ZnO} \mathrm{NW}$ also has to pass through the same steps required for the NWs grown using CVD methods. Swanwick et al. [13] reported symmetric and antisymmetric $\mathrm{ZnO} \mathrm{NW}$ near-UV sensor using low temperature hydrothermal growth without any further lithographic process after the growth; yet it required a careful design of the substrate and an extra sputtering step for $\mathrm{ZnO}$ layer. For the substitution of e-beam lithography process, numerous efforts have been made for the facile positioning of $\mathrm{ZnO} \mathrm{NW}$ on the premade electrode layer using different techniques such as direct printing [19] and dielectrophoresis [12]. These methods, 
however, often remain unwanted NWs at the unintended region of the substrate that lower the reliability of the process.

In this study, we propose laser-induced hydrothermal growth of $\mathrm{ZnO} \mathrm{NW}$ for the facile fabrication of $\mathrm{ZnO} \mathrm{NW}$ based UV sensor. For a conventional hydrothermal bulk growth of $\mathrm{ZnO} \mathrm{NW}$, a seeded substrate, either using $\mathrm{ZnO}$ nanoparticles [11] (NPs) or zinc acetate [20], is immersed in precursor solution that contains zinc ions and capping agents at elevated temperature for several hours to induce further anisotropic growth. Instead of heating the whole substrate, a focused laser has been utilized as a localized heater to raise the temperature locally and synthesize the NWs only at the desired position. By applying this process on two different points in sequence, $\mathrm{ZnO} \mathrm{NW}$ network consist of photoconductive NW junctions that bridges the gap between two pads can be readily produced without any additional lithographic process or complicated aligning techniques. We demonstrated that the resultant $\mathrm{ZnO} \mathrm{NW}$ junction shows clear response to the UV exposure, so that this process can be effectively utilized for the fabrication of $\mathrm{ZnO} \mathrm{NW}$ based sensor applications.

\section{Experimental}

2.1. $\mathrm{ZnO}$ Seed Preparation. $30 \mathrm{~mL}$ of $30 \mathrm{mM} \mathrm{NaOH}$ solution in ethanol is added slowly to $60 \mathrm{~mL}$ of $10 \mathrm{mM}$ zinc acetate dehydrate $\left(\mathrm{Zn}(\mathrm{OAc})_{2}\right)$ in ethanol. The reaction is continued for 2 hour at $60^{\circ} \mathrm{C}$ with a gentle stirring. The solution, after the complete reaction, is cooled to room temperature and stored in the refrigerator. The resultant synthesized $\mathrm{ZnO}$ NPs are $5-10 \mathrm{~nm}$ in diameter with quasi-spherical shape. For the seeding, the solution is dropped and spread on the target substrate, followed by washing out with fresh ethanol after several seconds before its complete dry. This coating step is repeated 3 4 times to ensure the complete coverage of $\mathrm{ZnO}$ NPs on the substrate. By coating $\mathrm{ZnO}$ NP layer on the substrate, the growth of $\mathrm{ZnO} \mathrm{NW}$ can be initiated from the adhered ZnO NPs ("seeds") without going through homogeneous nucleation step [21].

2.2. $\mathrm{ZnO}$ Precursor Solution Preparation. $25 \mathrm{mM}$ of zinc nitrate hexahydrate $\left(\mathrm{Zn}\left(\mathrm{NO}_{3}\right)_{2} \cdot 6 \mathrm{H}_{2} \mathrm{O}\right)$ and $25 \mathrm{mM}$ of hexamethylenetetramine (HMTA, $\mathrm{C}_{6} \mathrm{H}_{12} \mathrm{~N}_{4}$ ) are dissolved in DI water, and $5 \sim 7 \mathrm{mM}$ of polyethylenimine $\left(\mathrm{PEI}, \mathrm{C}_{2} \mathrm{H}_{5} \mathrm{~N}\right)$ is added for further hindrance of lateral growth. The solution is then heated at $95^{\circ} \mathrm{C}$ for $1 \mathrm{~h}$ and cooled to room temperature, whereas the white precipitates produced during the heating process are filtered out afterwards. The resultant precursor solution is transparent with yellowish color.

2.3. Laser-Induced Hydrothermal Growth of $\mathrm{ZnO} N W$. The overall process of laser-induced hydrothermal growth is depicted in Figure 1. The substrate initially goes through the seeding step as explained in Section 2.1. A small PDMS chamber is placed on top of the sample and filled with the precursor solution (details in Section 2.2). The opening of the chamber is then blocked with a cover glass to prevent any undesirable optical disturbances such as solvent evaporation.
The laser beam is focused on the sample through an optical system to generate a local temperature field at the sample.

$532 \mathrm{~nm}$ continuous wave (CW) Nd: YAG laser is used as the optical source, and a half-wave plate (HWP), together with a polarized beam splitter (PBS), is located after the mechanical shutter in order to control the laser power by rotating the polarization state and passing the vertical polarization only [22, 23]. Another 50:50 beam splitter is placed subsequently for the real-time measurement of the laser power. The laser beam is focused by using commercial objective lens (5X), and the laser power is adjusted in the range of $60 \sim 150 \mathrm{~mW}$ in order to find the optimum power that generates the proper temperature field for the $\mathrm{ZnO} N W$ growth. Two CCD modules are located on both sides to constitute a dual inverted microscope system for the in situ monitoring of the entire growth process.

\subsection{Fabrication of $\mathrm{ZnO} N W$ Based UV Sensor Using Laser- Induced Hydrothermal Growth. The electrode layer with two pads separated by sub-10 $\mu \mathrm{m}$ gap is deposited on a glass wafer in advance of the laser-induced hydrothermal growth process. The electrode layer not only provides the external interconnections for the electrical characterization but also acts as a uniform light-absorbing layer for the temperature rise. The $\mathrm{ZnO} \mathrm{NW}$ array is grown at the edges of two pads as shown in Figure 5(a). The laser power and growth time for each point is fixed to be $120 \mathrm{~mW}$ and $15 \mathrm{~min}$.}

2.5. Characterization. All the electrical characterizations are conducted in probe station having 4 axis micromanipulators in a dark faraday cage. The $I V$ curve and photocurrent time evolution are measured by a semiconductor analyzer, where the UV lamp has the peak power at $365 \mathrm{~nm}$ wavelength with $350 \mu \mathrm{W} / \mathrm{cm}^{2}$ power. The optical, SEM, and TEM images are acquired for characterization.

\section{Results and Discussion}

3.1. Laser-Induced Hydrothermal Growth of $\mathrm{ZnO} N W$. The growth of $\mathrm{ZnO} \mathrm{NWs}$ on gold coated glass wafer can be detected from CCD camera within several minutes when the laser power exceeds $\sim 60 \mathrm{~mW}$. We searched for the highest possible laser power for the synthesis of longer NW by generating extended temperature field. However, since the temperature rise happens in liquid environment, laser power that exceeds a certain threshold value induces a temperature that is higher than the boiling point of the solvent and creates a bubble which destroys the previously grown NW array. For the current optical configuration, a bubble easily appears at $150 \mathrm{~mW}$, and thus the laser power is fixed to be $120 \mathrm{~mW}$, unless stated otherwise, for sufficiently efficient but stable growth condition. Figure 2(a) shows the typical outcome of $\mathrm{ZnO}$ NW array produced by laser-induced hydrothermal growth process when the laser is focused at a single point using $5 \mathrm{X}$ objective lens for $30 \mathrm{~min}$. The temperature induced by the focused laser spot is the largest at the center due to the Gaussian profile of the laser beam, and thus the length of individual NW is the maximum at the middle of the array. The 


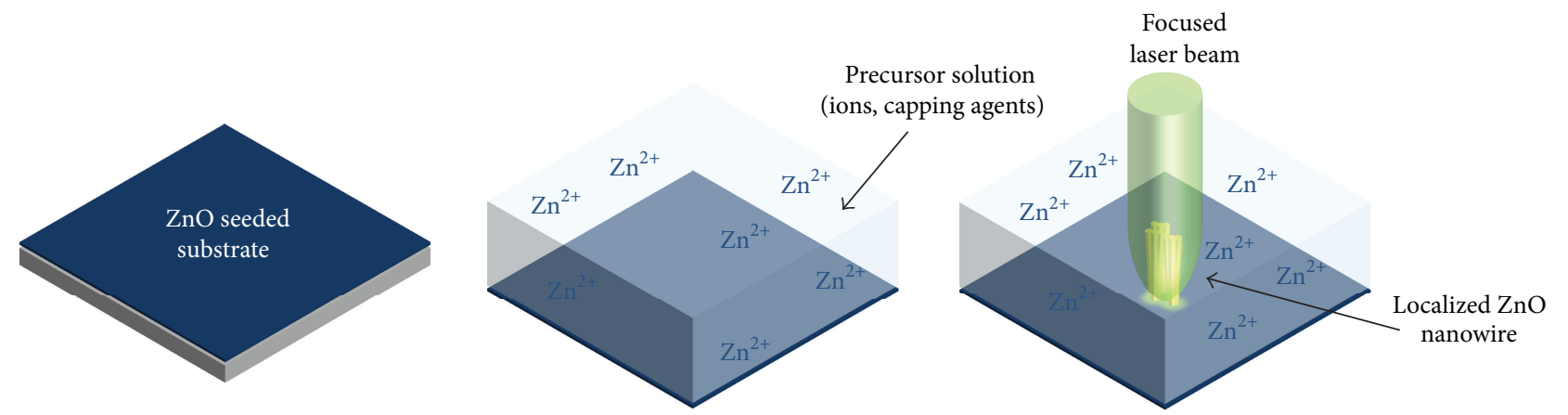

FIGURE 1: Schematic illustration of laser-induced hydrothermal growth process. The target substrate is seeded with $\mathrm{ZnO} \mathrm{NP}$, and immersed in the precursor solution. The laser is then focused on the sample surface to generate a confined temperature field to induce localized synthesis of $\mathrm{ZnO}$ NW.

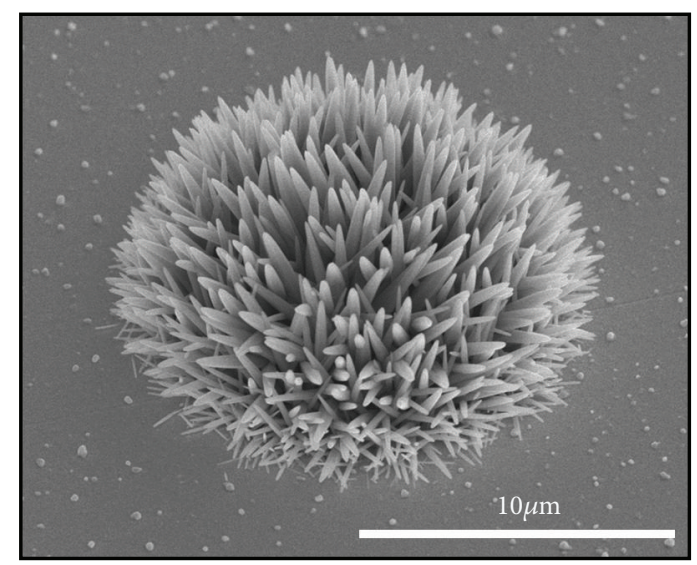

(a)

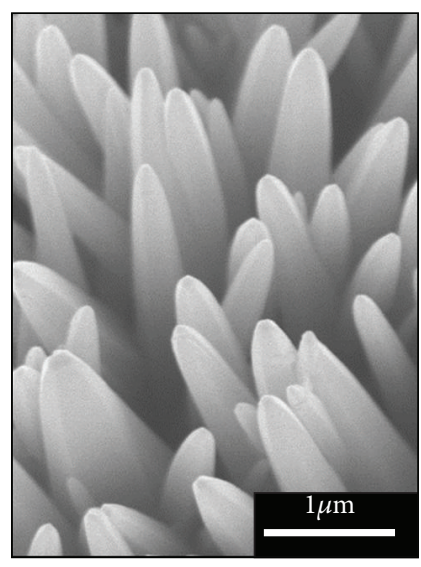

(b)

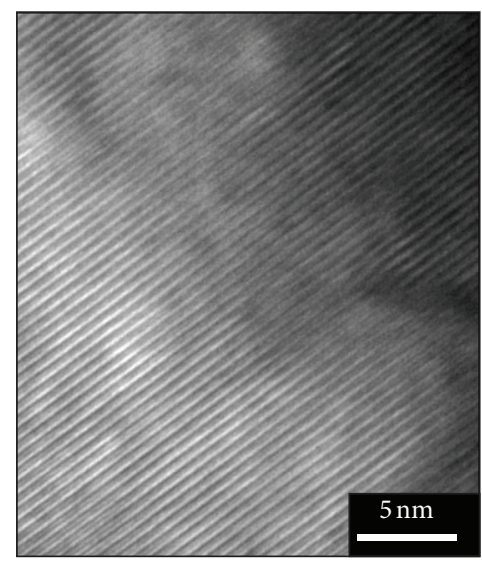

(c)

Figure 2: ZnO NW array synthesized using laser-induced hydrothermal growth at $120 \mathrm{~mW}$ power for 30 min using $5 \mathrm{X}$ objective lens. (a) SEM image of ZnO NW array synthesized by laser-induced hydrothermal growth. ZnO NW array consists of individual NWs facing radial direction with $\sim 8 \mu \mathrm{m}$ and length, is synthesized only at the laser spot. (b) SEM image of ZnO NW array with higher magnification. Distinct facets are observable, while the thickness of each NW is $200 \sim 400 \mathrm{~nm}$. (c) TEM image of a single ZnO NW, showing the lattice arrangement along $c$-axis.

size of the resultant ZnO NW array $(\sim 15 \mu \mathrm{m})$ is considerably larger than the estimated laser spot size $(\sim 10 \mu \mathrm{m})$ since the NWs at the edge are grown at more oblique angle to expand the lateral dimension of the array. These NWs grown at oblique angles are favorable for the fabrication of UV sensor as shown in Figure 5(a). From the magnified SEM image in Figure 2(b), distinct facets of $\mathrm{ZnO} \mathrm{NW}$ due to the wurtzite crystal structure can be confirmed. The lateral dimension of each NW is around $200 \sim 400 \mathrm{~nm}$, and its crystalline nature is noticeable in TEM image in Figure 2(c), showing its $c$-axis with the lattice constant of $0.52 \mathrm{~nm}$.

The dark-field optical image and the corresponding SEM image for different growth time $(5 \mathrm{~min}, 10 \mathrm{~min}, 15 \mathrm{~min}$, $20 \mathrm{~min}, 25 \mathrm{~min}$, and $30 \mathrm{~min}$ ) are shown in Figures 3(a) and $3(\mathrm{~b})$. The increase in the overall array size according to the elapsed growth time is apparent in both optical and SEM images. An interesting point is that the growth rate of laserinduced growth is much faster than that of the conventional bulk hydrothermal growth. For the conventional bulk growth using the same precursor solution, the growth speed is normally bounded to $\sim 2 \mu \mathrm{m} / \mathrm{hr}$. Recent studies on preferential growth [24] availed the growth speed to be as high as $\sim 5 \mu \mathrm{m} / \mathrm{hr}$ by adding different chemical compounds, but the growth rate of laser-induced growth easily exceeds $10 \mu \mathrm{m} / \mathrm{hr}$ and even reaches $20 \mu \mathrm{m} / \mathrm{hr}$ at higher laser power. Such rapid growth is considered to be originated from three different reasons [22]. Firstly, the overall concentration remains almost unchanged for the laser-induced hydrothermal growth process. For the conventional bulk hydrothermal growth, the length of NW grows asymptotically since the zinc precursor in the solution is constantly consumed in the course of growth process. As a result, the precursor solution has to be refreshed for the further growth. In contrary, the consumption of zinc precursor, in terms of total amount, is almost negligible for the laser growth, and thus the zinc precursor can be continuously supplied to the growth spot. Secondly, the zinc precursor is supplied from single direction in the case of conventional bulk growth; however, the zinc precursor can 


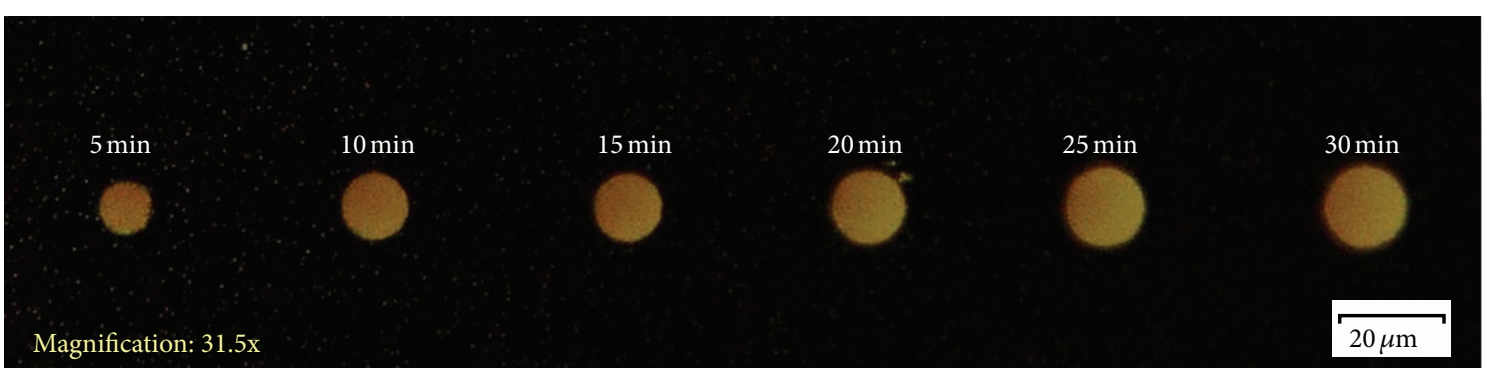

(a)
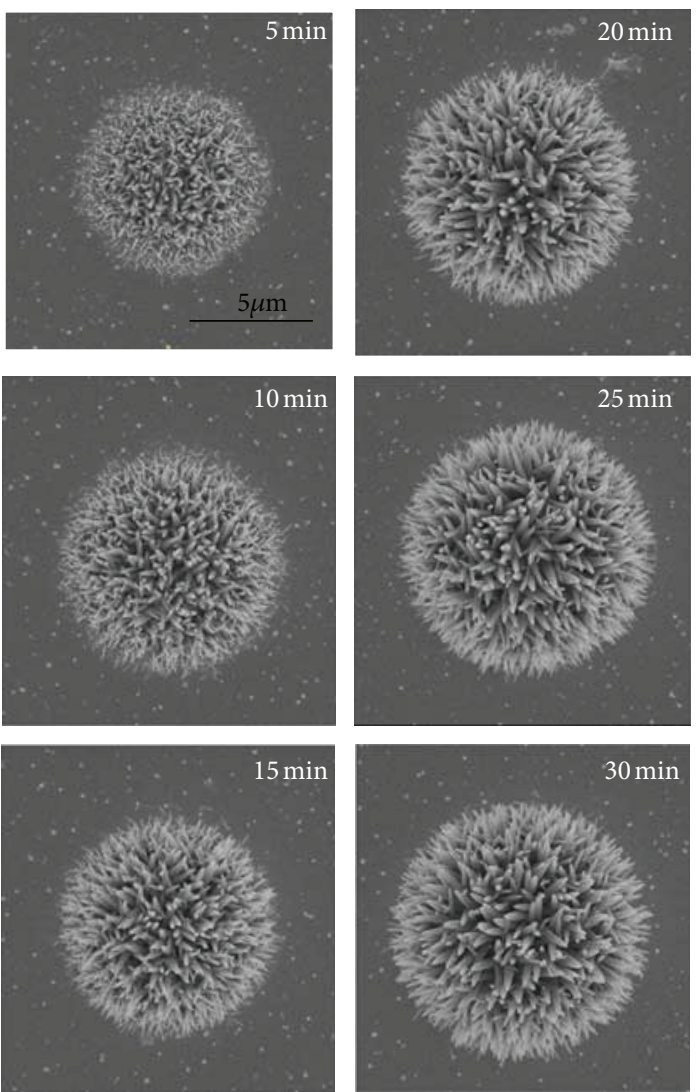

(b)

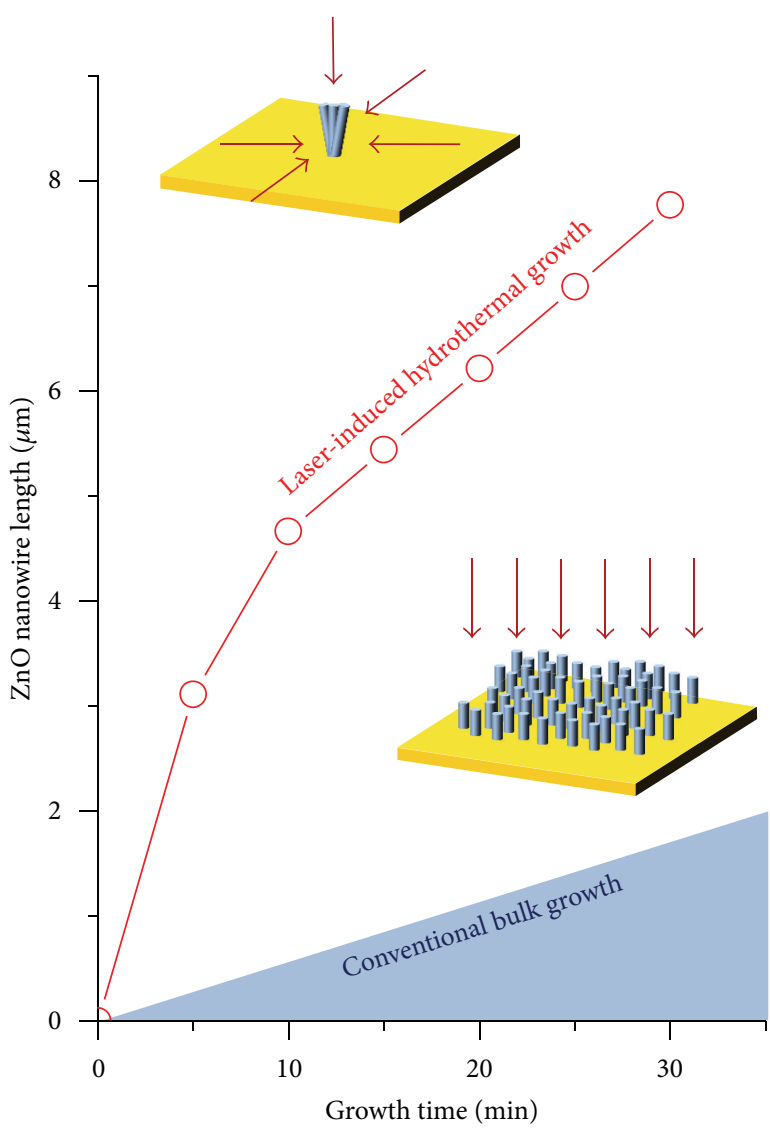

(c)

FIGURE 3: (a) Optical images of $\mathrm{ZnO} \mathrm{NW}$ array grown using laser-induced hydrothermal growth for $5 \mathrm{~min}, 10 \mathrm{~min}, 15 \mathrm{~min}, 20 \mathrm{~min}$, and $25 \mathrm{~min}$ and $30 \mathrm{~min}$ and (b) the corresponding SEM images. (c) Plot of the ZnO NW length at different growth time. It is noticeable that the growth rate of laser-induced hydrothermal process is much faster than the conventional bulk growth.

reach the growth spot from all radial direction for laserinduced growth as shown in the inset of Figure 3(c). Thirdly, the natural convection due to the temperature gradient might be also beneficial for the zinc precursor supply. These two properties of laser-induced growth-localized synthesis only at the desired position and rapid growth speed-are both advantageous for the facile fabrication of $\mathrm{ZnO} \mathrm{NW}$ based sensor [23].

3.2. $\mathrm{ZnO} \mathrm{NW}$ Based UV Sensor. It is well-known that $\mathrm{ZnO}$ NW changes its conductivity upon UV illumination [23, 25] as its working mechanism is illustrated in Figure 4.
A depletion region is formed at the surface of $\mathrm{ZnO} \mathrm{NW}$ in the dark because the oxygen molecules are adsorbed on the surface by capturing the free electrons in the ntype semiconductor. When the UV light that exceeds the bandgap of $\mathrm{ZnO}$ is illuminated to the $\mathrm{ZnO} \mathrm{NW}$, electronhole pairs are generated, and the holes migrate to the surface due to the potential slope. The oxygen molecule is then detached from the surface by capturing the hole, while the remaining electron contributes to the conductivity. The current therefore typically increases upon the UV exposure at a fixed voltage bias.

By applying laser-induced hydrothermal growth twice, the gap between two pads can be interconnected with UV 


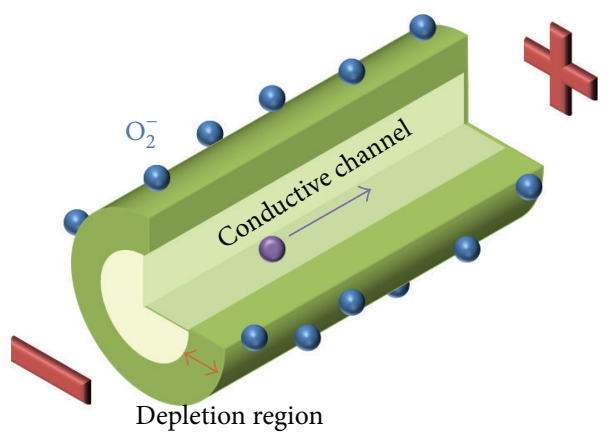

(a)

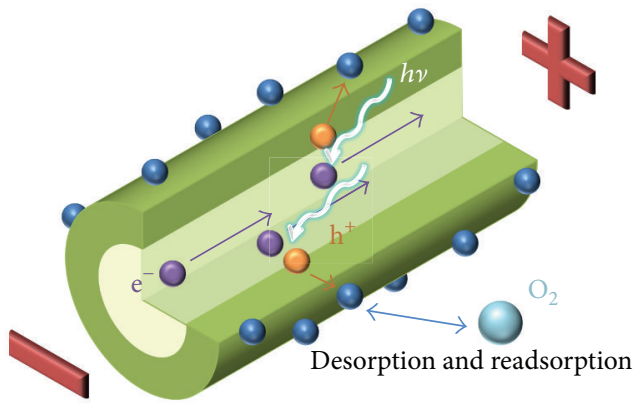

(b)

FIGURE 4: Schematics on the mechanism of ZnO NW based UV sensor. (a) In the dark, oxygen molecules are adsorbed on the surface of $\mathrm{ZnO} \mathrm{NW}$ by capturing the free electrons in n-type semiconductor. (b) Upon the UV illumination, electron-hole pairs are generated, and the oxygen molecules are detached from the surface by taking the holes. The remaining electrons contribute to the increase in conductivity.

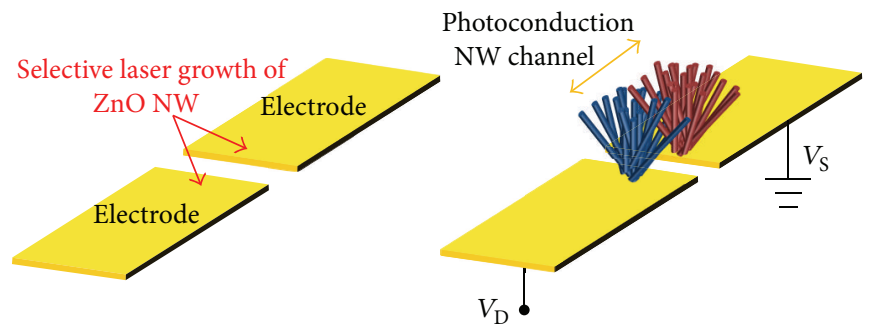

(a)

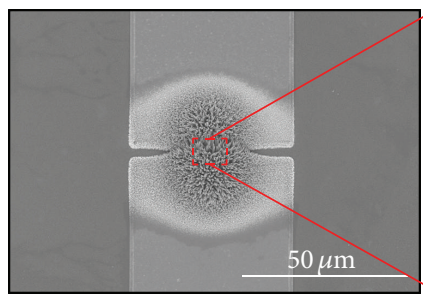

(b)

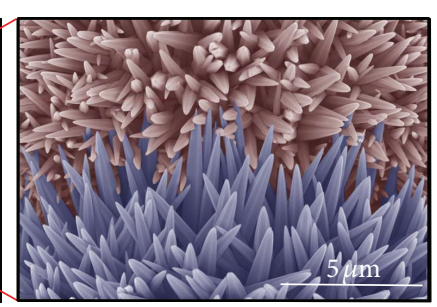

(c)

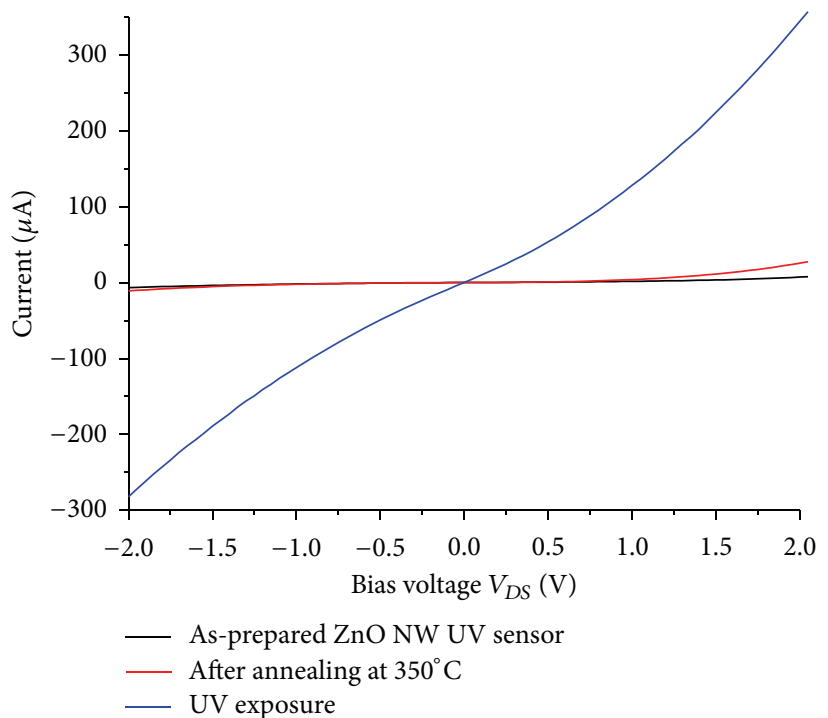

(d)

FIgURE 5: (a) Schematic of ZnO NW based UV sensor fabricated by laser induced hydrothermal growth. ZnO NW array is grown selectively at the two edges of the electrodes by applying laser-induced growth twice. The resultant $\mathrm{ZnO} \mathrm{NW}$ arrays form interconnecting ZnO NWs whose conductance changes upon UV illumination. (b) SEM image of the resultant ZnO NW based UV sensor (c) SEM image at higher magnification. It is noticeable that the NWs grown from different electrodes come into contact with each other. (d) $I V$ curves of as-prepared device, the device after annealing process and the same device upon UV illumination.

sensitive NW channel. The schematic diagram is shown in Figure 5(a), whereas the ZnO NW based UV sensor with the similar configurations can be found in the previous studies $[9,26]$ as well. It can be confirmed from the SEM image in Figure 5(b) that the grown $\mathrm{ZnO} \mathrm{NW}$ has sufficient length to bridge the gap between two pads. The high magnification SEM image in Figure 5(c) shows that the individual NWs are contacted to form photoconductive $\mathrm{ZnO} \mathrm{NW}$ channel. After the application of laser-induced hydrothermal growth, the device is annealed at $350^{\circ} \mathrm{C}$ for $10 \mathrm{~min}$ [27]. The $I V$ curves before and after the annealing process are plotted in Figure 5(d), and the device shows subtle difference upon the annealing process, possibly due to the polymer removal at the surface. While on the other hand, the current under the
UV illumination increases significantly by 2 to 3 orders of magnitude, which is comparable to the value acquired from single $\mathrm{ZnO}$ NW [25]. Such huge difference in current upon the UV illumination makes our device suitable for the UV detection.

The $I V$ curves in Figure 5(d) are not completely ohmic, but somewhat nonlinear and asymmetrical, showing diodelike characteristics. The electrical behavior of $\mathrm{ZnO} \mathrm{NW}$ in metal-semiconductor-metal configuration is still under debate, and previous studies have shown that the $\mathrm{ZnO} \mathrm{NW}$ can either form ohmic contact [25] or Schottky barrier [12], although the configurations are not completely the same. The origin of schottky barrier has been described by numerous explanations. The intrinsic property of $\mathrm{ZnO} \mathrm{NW}$ seems to 


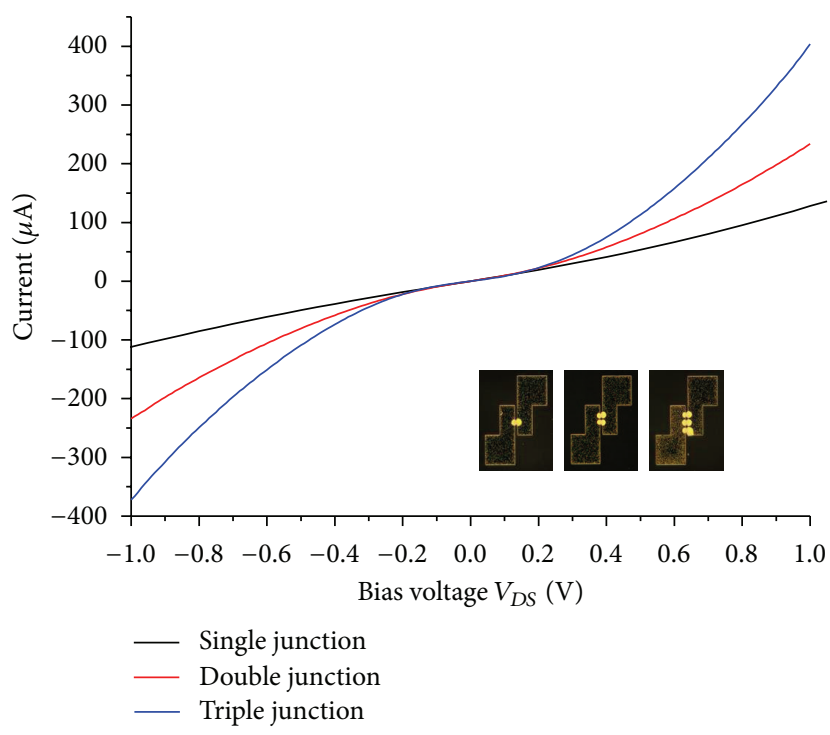

(a)

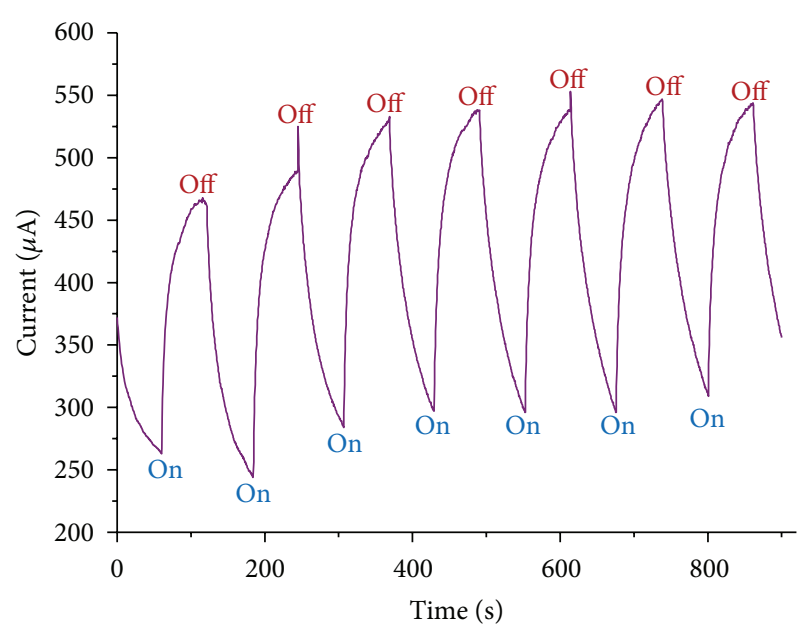

(b)

FIGURE 6: (a) IV curves of the ZnO NW based UV sensors with single, double, and triple junctions. The current increases almost linearly with the number of junctions. (b) Time evolution of the current when the UV illumination is switched on and off for every 60 seconds at a fixed bias voltage.

be responsible for the asymmetry in $I V$ curve in the first place, since the alternating zinc and oxygen layers of $\mathrm{ZnO}$ NW can influence the symmetry of $I V$ curve by providing dipole moment [12]. The process concerned for the fabrication of MSM configuration, such as electrode deposition or dielectrophoresis, can also contribute to the formation of the schottky barrier by providing different fixations, contact areas, and annealing conditions at both ends of the NW [16, 28]. It is reported that the properties of the schottky barrier is actually adjustable by intentional modulation of barrier height and width using different contacting metal layers for each end of the NW [28]. Furthermore, the transformation of ohmic contact into schottky barrier, or vice versa, is possible as well through various methods such as laser irradiation, bending, or annealing [29]. The electrical properties of the current device are also expected to be controllable by various methods to yield the optimum characteristics for sensing applications.

It is worth mentioning that the amount of photocurrent even at low bias voltage is relatively high for the UV sensor fabricated by laser-induced hydrothermal growth process due to the densely grown $\mathrm{ZnO} \mathrm{NW}$ at the growth spot. High photoresponse can be measured without any high-precision measurement system, so that the overall cost of the sensor can be reduced [19]. The photocurrent can be further adjusted by controlling the number of $\mathrm{ZnO} \mathrm{NW}$ array network junction digitally. Figure 6(a) shows the $I V$ curves upon the UV exposure when single, double, and triple $\mathrm{ZnO} \mathrm{NW}$ array junctions are synthesized between two pads by applying laserinduced growth 2, 4, and 6 times. The optical images of each device are shown in the inset. We can confirm that the current increases almost linearly with the number of the junctions.

Figure 6(b) shows the photocurrent of the UV sensor with triple junction when the UV illumination is switched on and off for every 60 seconds. The device presents clear response to the UV exposure; however, its saturation characteristics are not fully perceived. The rising and decay times are estimated from fitting a biexponential function. For the current device, the rising times are $4.2 \mathrm{~s}$ and $29.32 \mathrm{~s}$, while two decaying times are $4.41 \mathrm{~s}$ and $38.6 \mathrm{~s}$. These values are comparable to the similar device fabricated with a dielectrophoretic method, [30] and much smaller than the recovery time of the UV sensor based on $\mathrm{ZnO}$ thin film [19]. The high magnified SEM image in Figure 5(c) shows that the NWs grown from two pads almost agglomerate and start to lose their properties as individual NWs. Therefore, the response and recovery time are expected to be reduced further by shortening the growth time.

\section{Conclusion}

We introduced laser-induced hydrothermal growth process in order to grow $\mathrm{ZnO} \mathrm{NW}$ array rapidly at a desire position without any high temperature or vacuum environment. By controlling the laser power and irradiation time, $\mathrm{ZnO} \mathrm{NW}$ arrays were easily integrated to the premade electrode layers to constitute photoconductive channel by bridging the gap between two electrode pads to demonstrate UV sensor. The resultant UV sensor displayed clear response to the UV exposure, showing that this process has a high potential for the facile fabrication of low-cost $\mathrm{ZnO} \mathrm{NW}$ based UV sensor.

\section{Acknowledgments}

This work is supported by National Research Foundation of Korea (NRF) (Grant nos. 2012-0008779, 2012-0003722), Global Frontier R\&D-Program on Center for Multiscale 
Energy System (Grant no. 2012-054172) under the Ministry of Science, ICT \& Future, Korea, and the cooperative R\&D Program (Grant no. B551179-10-01-00) on the Korea Research Council Industrial Science and Technology.

\section{References}

[1] M. H. Huang, S. Mao, H. Feick et al., "Room-temperature ultraviolet nanowire nanolasers," Science, vol. 292, no. 5523, pp. 1897-1899, 2001.

[2] H. W. Kang, J. Yeo, J. O. Hwang et al., "Simple $\mathrm{ZnO}$ nanowires patterned growth by microcontact printing for high performance field emission device," Journal of Physical Chemistry C, vol. 115, no. 23, pp. 11435-11441, 2011.

[3] M. Law, L. E. Greene, J. C. Johnson, R. Saykally, and P. Yang, "Nanowire dye-sensitized solar cells," Nature Materials, vol. 4, no. 6, pp. 455-459, 2005.

[4] S. Xu, Y. Qin, C. Xu, Y. Wei, R. Yang, and Z. L. Wang, "Selfpowered nanowire devices," Nature Nanotechnology, vol. 5, no. 5, pp. 366-373, 2010.

[5] X. Wang, J. Zhou, J. Song, J. Liu, N. Xu, and Z. L. Wang, "Piezoelectric field effect transistor and nanoforce sensor based on a single ZnO nanowire," Nano Letters, vol. 6, no. 12, pp. 27682772, 2006.

[6] Y. Zhang, K. Yu, D. Jiang, Z. Zhu, H. Geng, and L. Luo, "Zinc oxide nanorod and nanowire for humidity sensor," Applied Surface Science, vol. 242, no. 1-2, pp. 212-217, 2005.

[7] S. N. Das, J. P. Kar, J. H. Choi, T. I. Lee, K. J. Moon, and J. M. Myoung, "Fabrication and characterization of $\mathrm{ZnO}$ single nanowire-based hydrogen sensor," Journal of Physical Chemistry C, vol. 114, no. 3, pp. 1689-1693, 2010.

[8] A. Choi, K. Kim, H. Jung, and S. Y. Lee, "ZnO nanowire biosensors for detection of biomolecular interactions in enhancement mode," Sensors and Actuators B, vol. 148, no. 2, pp. 577-582, 2010.

[9] A. Menzel, K. Subannajui, F. Güder, D. Moser, O. Paul, and M. Zacharias, "Multifunctional ZnO-nanowire-based sensor," Advanced Functional Materials, vol. 21, no. 22, pp. 4342-4348, 2011.

[10] C. S. Lao, M. C. Park, Q. Kuang et al., "Giant enhancement in UV response of $\mathrm{ZnO}$ nanobelts by polymer surfacefunctionalization," Journal of the American Chemical Society, vol. 129, no. 40, pp. 12096-12097, 2007.

[11] L. E. Greene, M. Law, J. Goldberger et al., "Low-temperature wafer-scale production of $\mathrm{ZnO}$ nanowire arrays," Angewandte Chemie-International Edition, vol. 42, no. 26, pp. 3031-3034, 2003.

[12] O. Harnack, C. Pacholski, H. Weller, A. Yasuda, and J. M. Wessels, "Rectifying behavior of electrically aligned $\mathrm{ZnO}$ nanorods," Nano Letters, vol. 3, no. 8, pp. 1097-1101, 2003.

[13] M. E. Swanwick, S. M. L. Pfaendler, A. I. Akinwande, and A. J. Flewitt, "Near-ultraviolet zinc oxide nanowire sensor using low temperature hydrothermal growth," Nanotechnology, vol. 23, no. 34, Article ID 344009, 2012.

[14] N. Liu, G. Fang, W. Zeng et al., "Direct growth of lateral $\mathrm{ZnO}$ nanorod UV photodetectors with schottky contact by a single-step hydrothermal reaction," ACS Applied Materials and Interfaces, vol. 2, no. 7, pp. 1973-1979, 2010.

[15] Y. C. Kong, D. P. Yu, B. Zhang, W. Fang, and S. Q. Feng, "Ultraviolet-emitting $\mathrm{ZnO}$ nanowires synthesized by a physical vapor deposition approach," Applied Physics Letters, vol. 78, no. 4, pp. 407-409, 2001.

[16] Z. M. Liao, J. Xu, J. M. Zhang, and D. P. Yu, "Photovoltaic effect and charge storage in single $\mathrm{ZnO}$ nanowires," Applied Physics Letters, vol. 93, no. 2, Article ID 023111, 3 pages, 2008.

[17] K. H. Lee, S. W. Lee, R. R. Vanfleet, and W. Sigmund, "Amorphous silica nanowires grown by the vapor-solid mechanism," Chemical Physics Letters, vol. 376, no. 3-4, pp. 498-503, 2003.

[18] R. S. Wagner and W. C. Ellis, "Vapor-liquid-solid mechanism of single crystal growth," Applied Physics Letters, vol. 4, no. 5, pp. 89-90, 1964.

[19] S. Bai, W. Wu, Y. Qin, N. Cui, D. J. Bayerl, and X. Wang, "Highperformance integrated $\mathrm{ZnO}$ nanowire UV sensors on rigid and flexible substrates," Advanced Functional Materials, vol. 21, no. 23, pp. 4464-4469, 2011.

[20] L. E. Greene, M. Law, D. H. Tan et al., "General route to vertical $\mathrm{ZnO}$ nanowire arrays using textured $\mathrm{ZnO}$ seeds," Nano Letters, vol. 5, no. 7, pp. 1231-1236, 2005.

[21] S. Xu and Z. L. Wang, "One-dimensional ZnO nanostructures: solution growth and functional properties," Nano Research, vol. 4, no. 11, pp. 1013-1098, 2011.

[22] J. Yeo, S. Hong, M. Wanit et al., "Rapid, one-step, digital selective growth of $\mathrm{ZnO}$ nanowires on 3D structures using laser induced hydrothermal growth," Advanced Functional Materials, vol. 23, no. 26, pp. 3316-3323, 2013.

[23] S. Hong, J. Yeo, W. Manorotkul et al., "Digital selective growth of a $\mathrm{ZnO}$ nanowire array by large scale laser decomposition of zinc acetate," Nanoscale, vol. 5, no. 9, pp. 3698-3703, 2013.

[24] C. Xu, P. Shin, L. Cao, and D. Gao, "Preferential growth of long $\mathrm{ZnO}$ nanowire array and its application in dye-sensitized solar cells," Journal of Physical Chemistry C, vol. 114, no. 1, pp. 125-129, 2010.

[25] C. Soci, A. Zhang, B. Xiang et al., "ZnO nanowire UV photodetectors with high internal gain," Nano Letters, vol. 7, no. 4, pp. 1003-1009, 2007.

[26] Y. Li, F. D. Valle, M. Simonnet, I. Yamada, and J. J. Delaunay, "High-performance UV detector made of ultra-long $\mathrm{ZnO}$ bridging nanowires," Nanotechnology, vol. 20, no. 4, Article ID 045501, 2009.

[27] S. H. Ko, D. Lee, H. W. Kang et al., "Nanoforest of hydrothermally grown hierarchical $\mathrm{ZnO}$ nanowires for a high efficiency dye-sensitized solar cell," Nano Letters, vol. 11, no. 2, pp. 666671, 2011.

[28] C. S. Lao, J. Liu, P. Gao et al., " $\mathrm{ZnO}$ nanobelt/nanowire schottky diodes formed by dielectrophoresis alignment across au electrodes," Nano Letters, vol. 6, no. 2, pp. 263-266, 2006.

[29] Y. Hu, Y. Chang, P. Fei, R. L. Snyder, and Z. L. Wang, "Designing the electric transport characteristics of $\mathrm{ZnO}$ micro/nanowire devices by coupling piezoelectric and photoexcitation effects," ACS Nano, vol. 4, no. 2, pp. 1234-1240, 2010.

[30] L. Guo, H. Zhang, D. Zhao et al., "High responsivity $\mathrm{ZnO}$ nanowires based UV detector fabricated by the dielectrophoresis method," Sensors and Actuators B, vol. 166-167, pp. 12-16, 2012. 

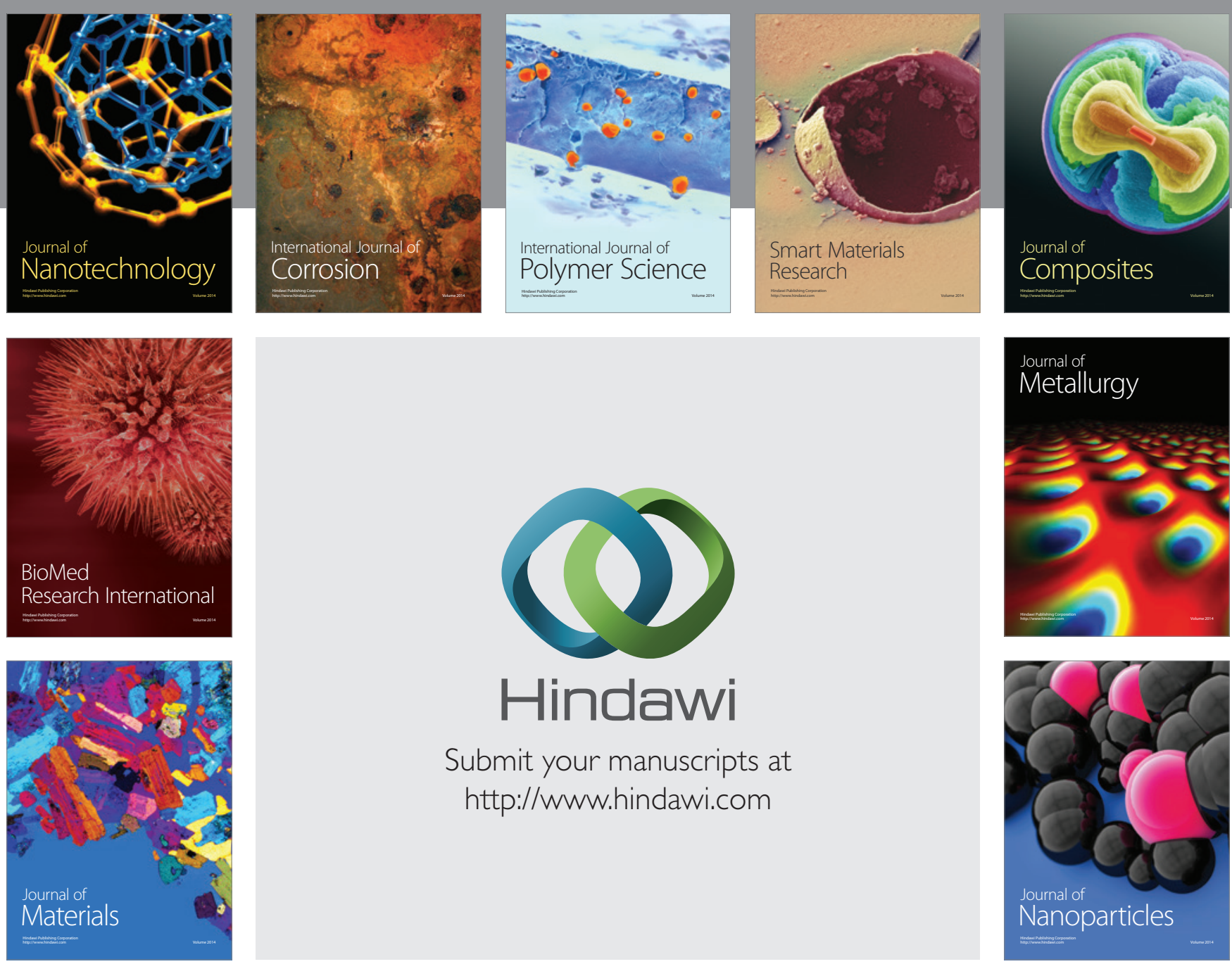

Submit your manuscripts at http://www.hindawi.com
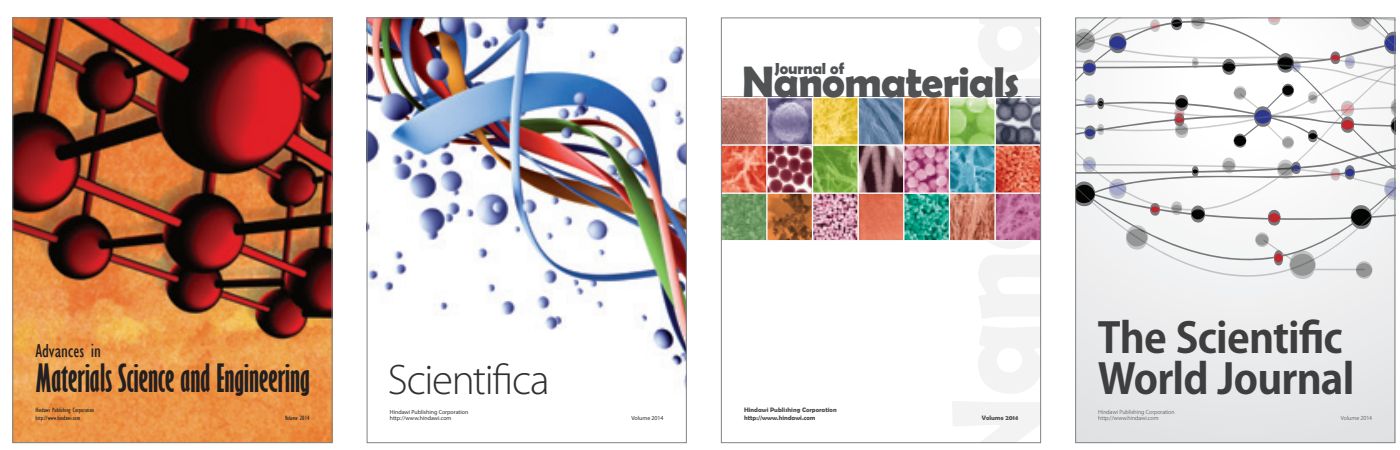

\section{The Scientific World Journal}
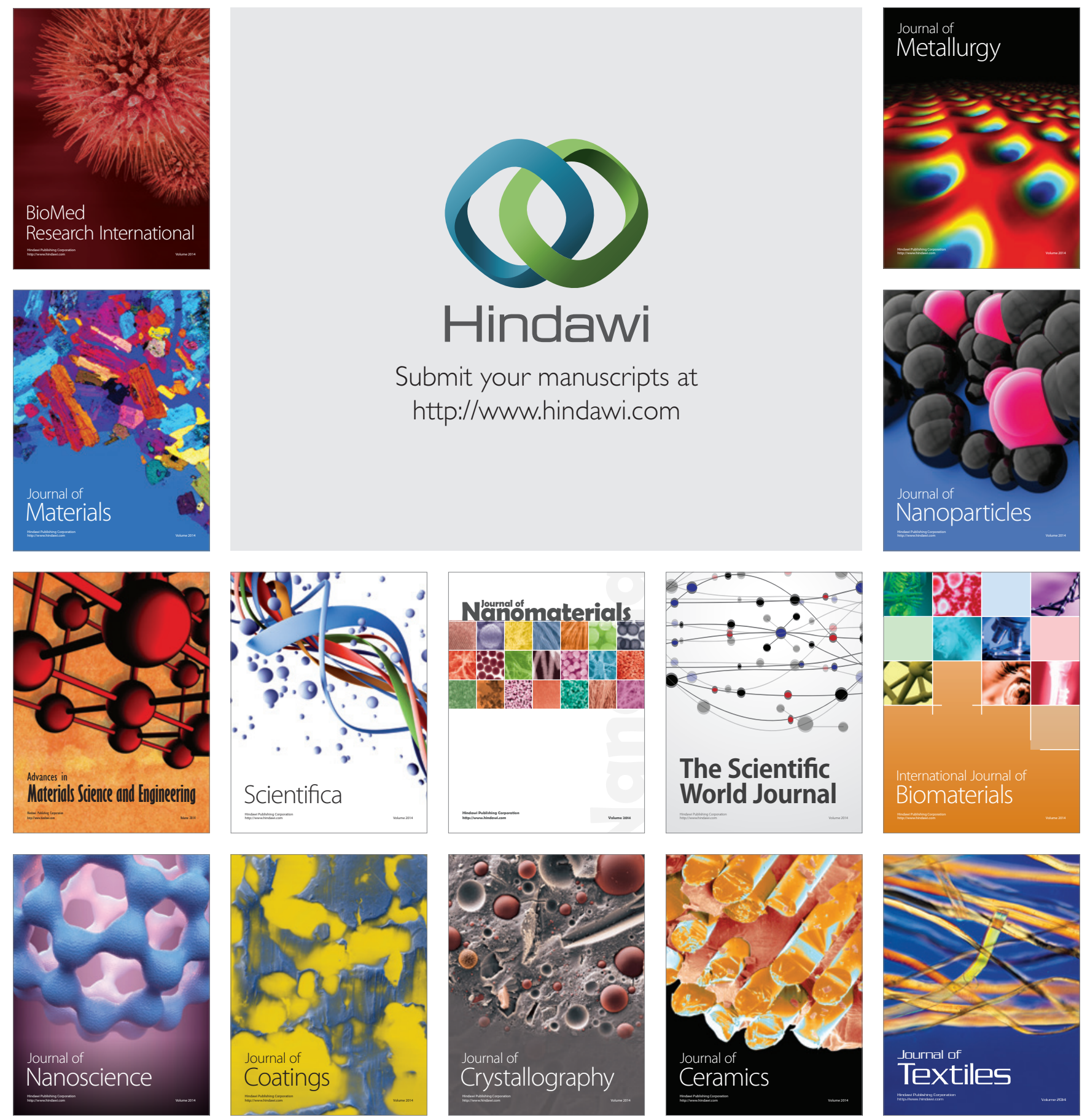